\title{
$\mathfrak{I} \mathfrak{n} \mathfrak{h} \mathfrak{a} \mathfrak{l} \mathbf{t}$.
}

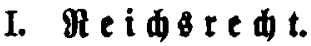

sert. Seite

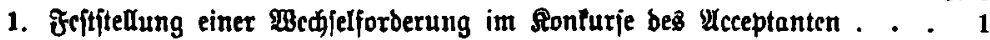

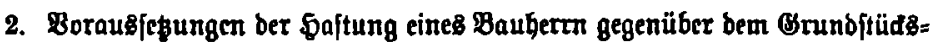
nadbarn für ben Sdjaben, ber baburd entfteht, baß bie etforberlidien Siderungsmabirrgeln nidjt getroffen find .

3. Mengen bon Єaden ober Maren im Ginne ber Rarifnumtmer $4 \mathrm{a}$ bes

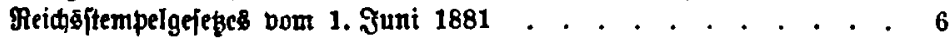

4. Einflus einer im ₹radtbriefe enthaltenen unridtigen Bezeidnung ber

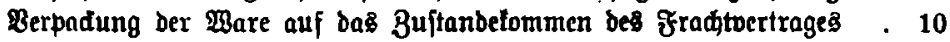

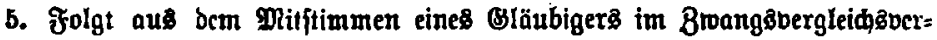

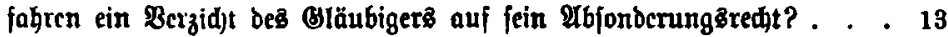

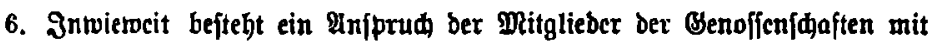

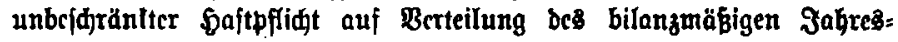
geminne\&? . . . . . . . . . . . . . . . . . . . 18

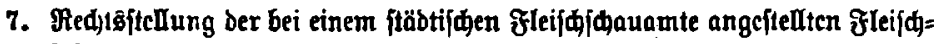
befdauer . . . . . . . . . . . . . . . . . . . . . 20

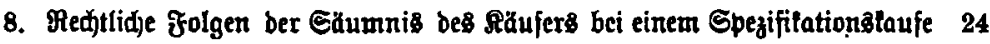

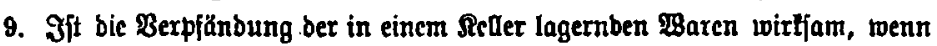
Der Berbfäuber einen ber vorbandenen Selleridglüffel für fï bebält? . 31

110. Einflup bes Strafurteilcs auf bic Entfaeibung bes Cibilridjters über bie Mrgreß̈llage ber Berufggenoffenfdaft . •. . . . . . . . . . 37

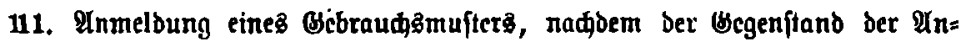


nt.

nelbung zur Crtcilung cines Batentę angemclbet war. Offenlunbige Benupung cines Drobelles. . . . . . . . . . . . . . . . 38

12. Erfindung und Erfinderredt bor ber Alnmeloung und Ertcilung eines Batentes.

13. Feftiftellung besె 8uftande? beanftandeter

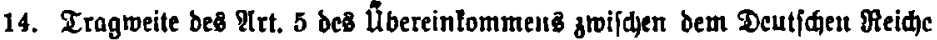

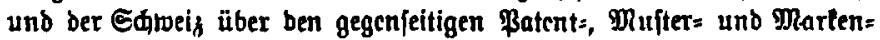
f(pub bom 13. शlpril 1892

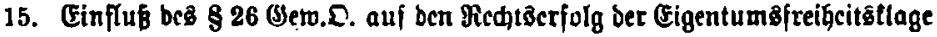

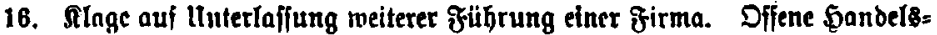
gejelljjaft . . . . . . . . . . . . . . . . . . . . .

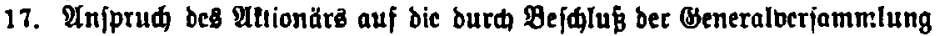
feitgefegte Dibibende.

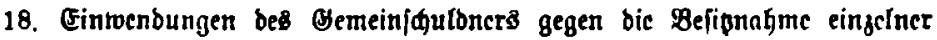
Bermögens̄itüde burd ben Sontursvertvalter

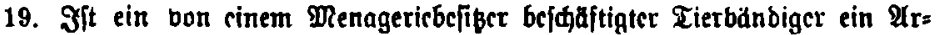

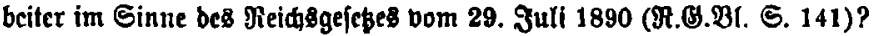

20. Eigentumälage auf \$ernusigabe gejtob!ener Эngaberpapiere. Buter Braube beim Eriverbc

21. Berchnung ber Eibilpenfion eines im Cibilbienfile angejteuten Militär= autwätters . . . . . . . . . . . . . . . . . . . . .

22. Berjäbrung ber Rlage gegen ben Bettäufer, ber bie Besuäbr für bie

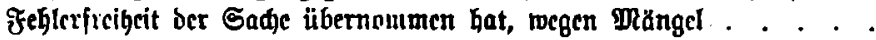

23. Rlage bes Ronlurosberwalters einer Sommanditgejelfijaft gegen ben Summanditiften auf Einzablung ber Einlage . . . . . . . . .

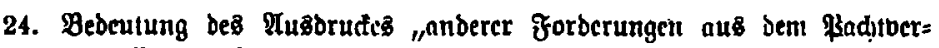

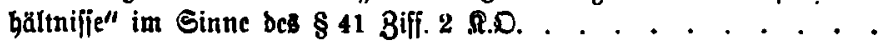

25. Anjprud des Sionlurşverwalters aus einer zu Bunjten bez Bemein= ¡ఘulbners erfolgten Sdjulbübernaljmc .

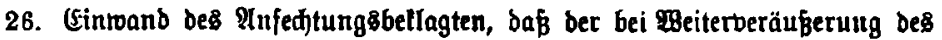

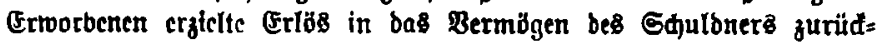
gevö̆brt jei .

27. Anfectung ber Ceffion einer forberung. Fiändung ber abgetretenen forderung. Simuliente Geffion.

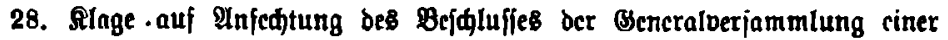

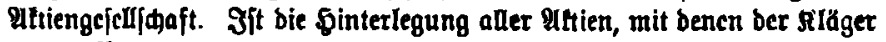
in ber Berjammlung gejtimat bat, Borausjekung ber ßlagc? . . -

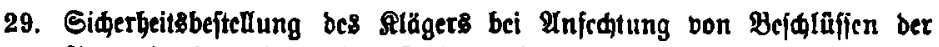

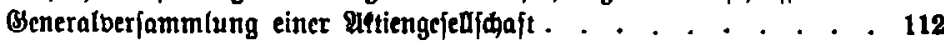

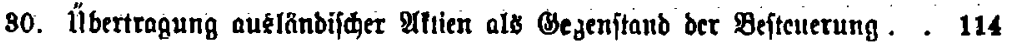




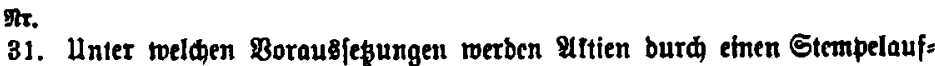
bruff zu neuen Altien im Sinne bes Meidsftempelgejeģes? . . . .

. Buflänbigleit für Gtreitigleiten ztwijळen einer Ortolranlenlafic unb eincm Arbeitgeber tiber beffen Beitragspflidjt

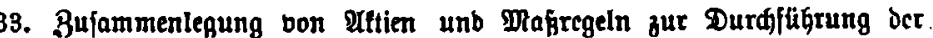
Bujamnenlegung. . . . . . . . . . . . . . . . . . .

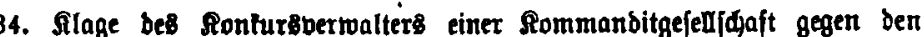
Sommanditiften auf Einzablung ber Einlage . . . . . . . . .

35. Werpfänoung ber Stammaltien einer Rübenzuderfabril bei Befdränfung

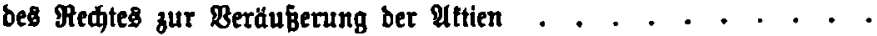

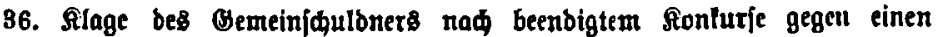
früberen fonlurbgläubiger auf Bahlung einer Begenforberung gegen

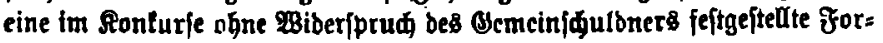
berung

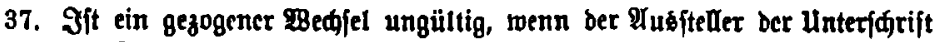
bie Mlaujel "ofgne Betoäbr" beigefügt hat? . . . . . . . . . .

\section{Scmeines Pedt.}

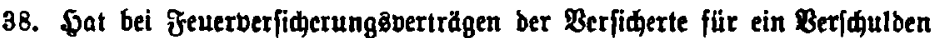
feinç Bertreter einzujtehen? . . . . . . . . . . . . . . 149

39. Sit bie Robizillartlaujel aud gegen einell postumas wirljam? . . . 151

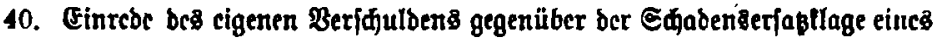
impubes infantia maior . . . . . $\cdot \cdot \cdot \cdot \cdot \cdot \cdot \cdot \cdot \cdot \cdot \cdot \cdot \cdot \cdot$

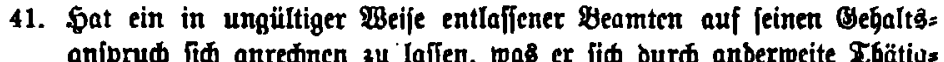
leit etworben bat? . . . . . . . . . . . . . . . . . 160

12. Bebeutung bes Becboteङ Der Betăußerung bon Dotalfaçen . . . . 161

43. Boraubjeģungen ber (3ültigleit eincs Arboptionsbertages. - Einjeitiger Füdtritt . . . . . . . . . . . . . . . . . . . . . .

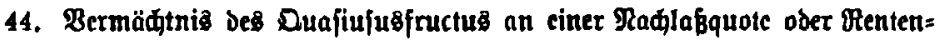
vermädtnis. . . . . . . . . . . . . . . . . . 168

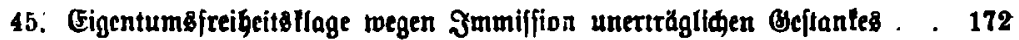

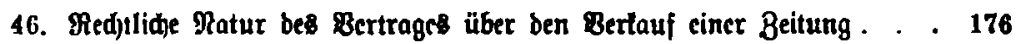

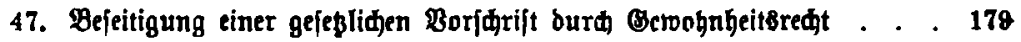


Tr.

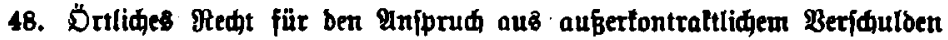
gegen einer Dritten . . . . . . . . . . . . . . . . . . 181

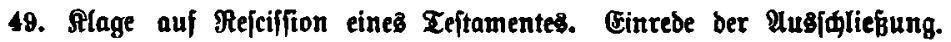
Replit ber $\mathfrak{A}$ rglifit

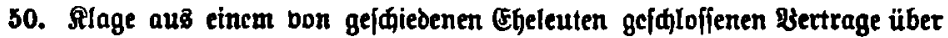

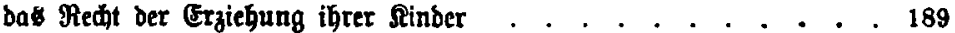

b1. Infed)lung cines geridtlid abgejめloffenen Brozeibergleiđ)es wegen

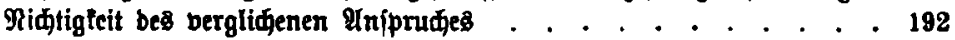

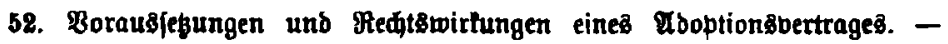
Duajipupillarjubftitution . . . . . . . . . . . . . . . . 192

\section{Breußifde Redt.}

53. Rlage auf Sdabenserlał gegen einen Baukerrn

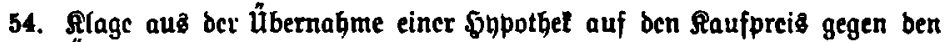
übernchmer . . . . . . . . . . . . . . . . . . .

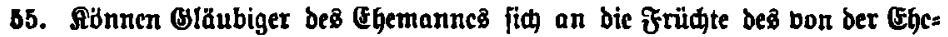

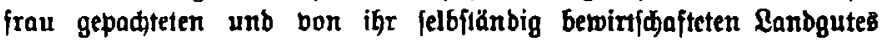
balten?

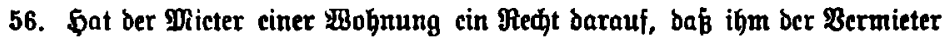

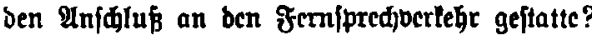

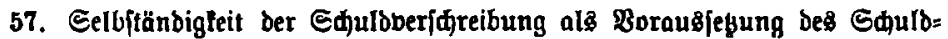
ber|dreibungâfempcls . . . . . . . . . . . . . . . . .

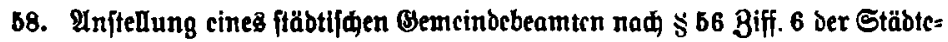

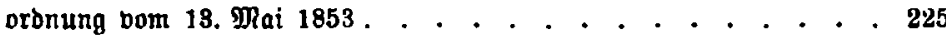

69. Beređnung ঠer Civilpenfion eines Mlilitäranwärters. . . . . . . 235

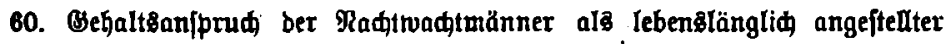
Bemeindebeamter .

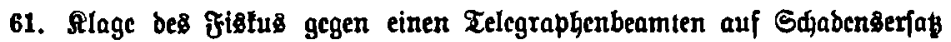

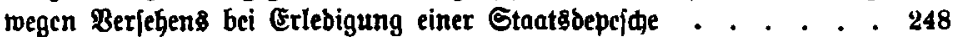

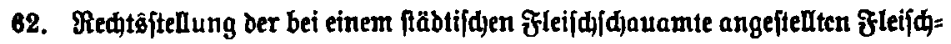
bejdauex

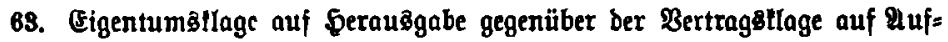
laffung . $\cdot \cdot \cdot \cdot \cdot \cdot \cdot \cdot \cdot \cdot \cdot \cdot \cdot \cdot \cdot \cdot \cdot \cdot \cdot \cdot \cdot \cdot \cdot \cdot \cdot \cdot \cdot \cdot$

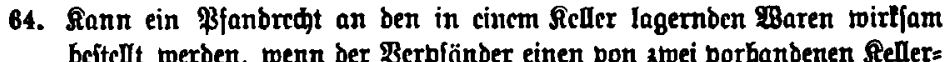

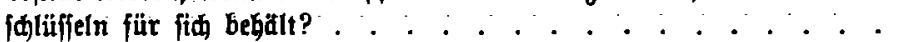




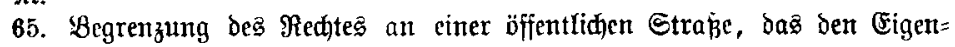

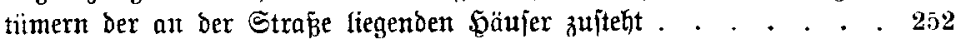

66. Wilage einer Stadtgemeinde gegen Den Fißluts auf Eritattung ber non ihr

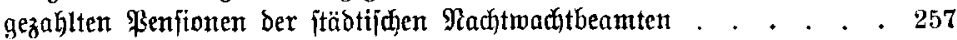

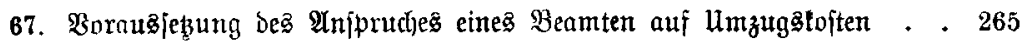

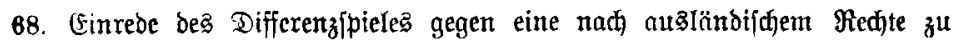
beurteilende Forberung. . . . . . . . . . . . . . . . .

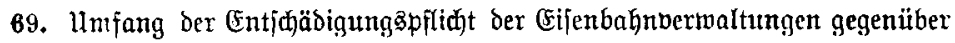

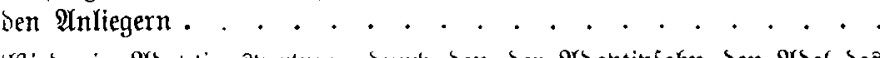

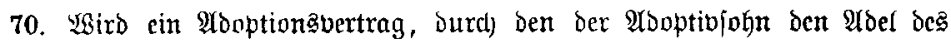
Idoptibbaters erfalten foll, ungültig, menn bie landeăherrlifje (Ge= nehmigung berjagt twirb?

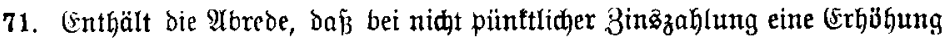

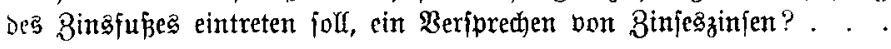

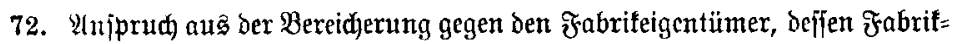

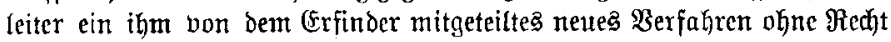
angementet hat

73. Ṡerjährumg ber Fordenungen ber Biegelmeifter auf Bahlung bes rüđt=

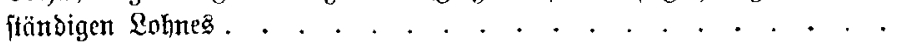

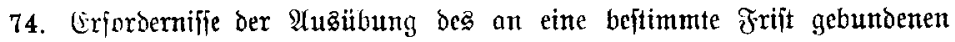
\$icderfaufärchtes . . . . . . . . . . . . . . . . . .

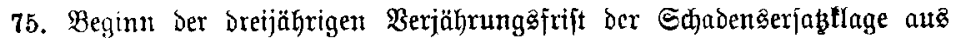

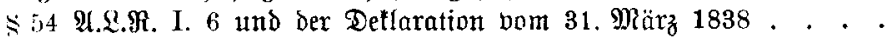

76. Sertragşmäp̈ige baftung be getretenen Forberung ....................

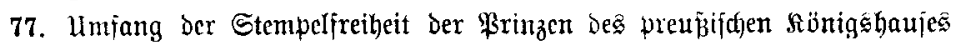

78. Stempelabgabe jür Qteferungşberträge über \$iengen von ธadjen . .

79. Unjtellung einer $\mathfrak{L} e \mathfrak{h r c r i n}$ an einer Mäbdjenidhule unter ber Bebingung, ১aß̉ fic fich nidht verhcirate . . . . . . . . . . . . . . . 298

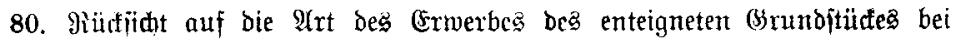
Tejtiełung ber Enteignungşumme. . . . . . . . . . . . . 305

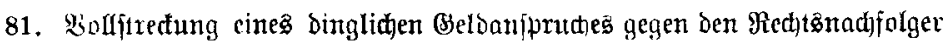

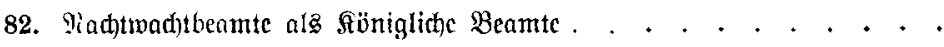

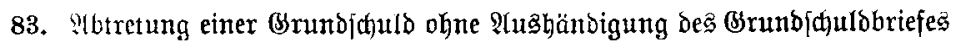

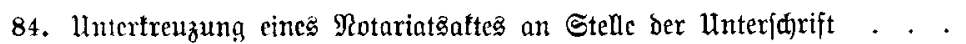

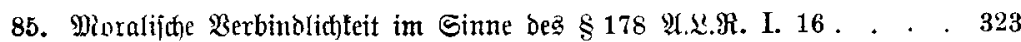

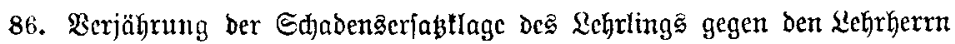

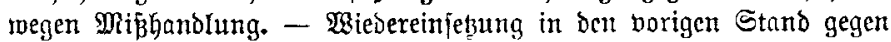
bie vollendcte Berjährung . 
Th.

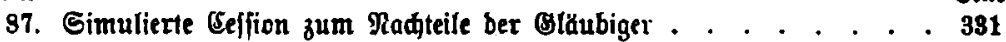

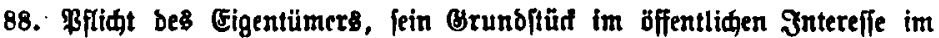
Stande zu erbalten . . . . . . . . . . . . . . . . . . 331

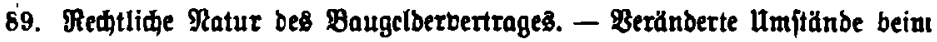
Esemfel ber Berfon bes Baukerrn .............. 336

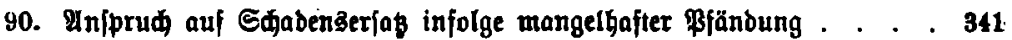

91. Sllage eincs Eingebfarrten, ber gleidzzeitig Rirdenpatron ift, auf ofrei= laffung bon einer \$ithenfteucr . . . . . . . . . . . . . . . 34

92. Einwand ber Berzeibung im Ehefdeibungsprozeffe. - Reservatio mentalig . . . . . . . . . . . . . . . . . . . .

93. Bertrag bes \$auptes cinet ftandeshertiden familie über feinen unb feiner Nadfolger Werzidt auf bie Steuerfreigeit . . . . . . . . 348

94. Eelfion einer Sypotbel gegen Entgelt . . . . . . . . . . . . 950

96. 2ffterberpođtung von Teilen eine\& Lanogutcs . . . . . . . . . 354

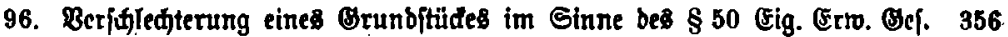

\section{Rbeintide Redt.}

97. Findet $\mathfrak{A}$ rt. 1184 Code civil aud auf Betgletife Alnwendung? . . . 361

98. Rectllidbe Bebeutung ber $\mathfrak{X}$ (uflaffung bes Miteigentumes an einem

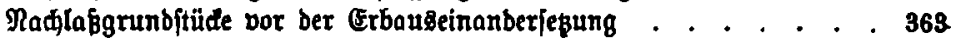

99. Pfänbung bes Mehtes bes Mieterş auf Benuß̧ung ber gemieteten Mäum= lidleiten . . . . . . . . . . . . . . . . . 36T

\section{Brozepredt.}

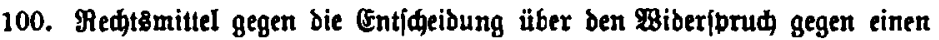
Irrejtbejdlus

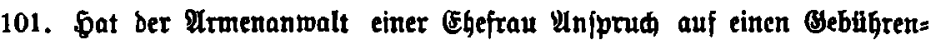
und A्A

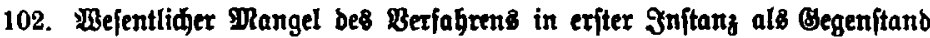

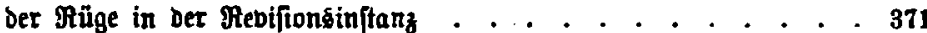

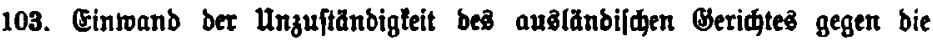

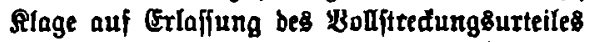

104. Ift Dem Q̂Antrage bes mit ber Eigentumbllage auf ferausgabe eines Grunbitüdes belangten Bellagten auf Tu\&fepung bet Bethandling bis 
"sr.

zur Etlebigung ber bon bem Intragiteller gegen ben Eigentum sltäger erbobenen Bertragsillage auf Muflafiung ftattzugeben? . . . . . 973

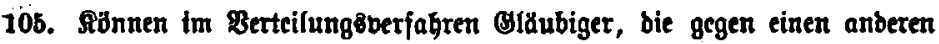
Bläubiger getlagt baben, mit ber Betufung gegencinanber auftreten? 376

106. Sann auf bic Befolgung ber friftbot/Arift bes $\$ 809$ थ6f. 2 c..\$3.D. (\$ 267 C.\$.D.) verzidtet werber? . . . . . . . . . . . 378

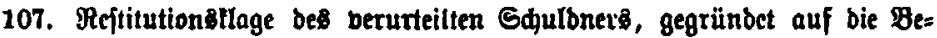
hauptung, baß ber Mçtitutionsbetlagte zur \$lagerhebung nimt legiti= miert gerocjen joi . . . . . . . . . . . . . . . .. . .

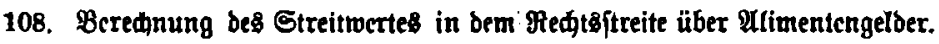
- Bejdrwerde . . . . . . . . . . . . . . . . . . . 382

109. Reftitutionstlage gegen cine Cintragung in bic Ronlur $\$$ tabelle . . 386

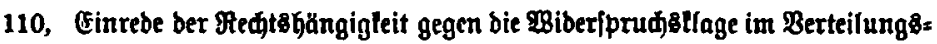
berfakten . . . . . . . . . . . . . . . . . 990

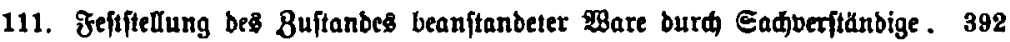

112. Bujtänbigleit für bie Entideibung über bie Fedt Borbertrageş beh̆

113. Albgrenzung ber Buläjfïgleit eines Ieilurteiles . . . . . . . . 996

114. Weitere Befdjwerbe gegen ben Bejdlufb, ourd ben auf erhobene $B=$

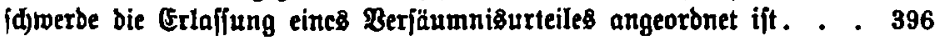

115. Eintonbungen bes Bemeinjoulbners gegen bie Befïnabme einzelner

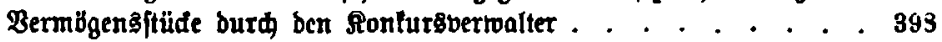

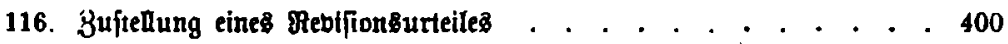

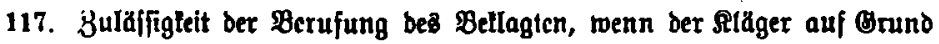
einer $\mathfrak{e}$ ufrednungseinrebe abgerwiejen ift . . . . . . . . . . 403

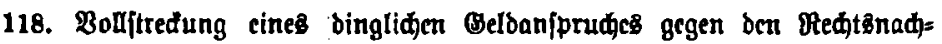
folget . . . . . . . . . . . . . . . . . 406

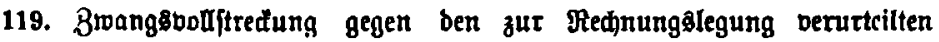
હduloner. . . . . . . . . . . . . . . . . . . . 406

120. Beginn ber 3 wangşbouftredung zur Erwirtung bon unterlaffungen . 409

121. Jit \$657 (5.P.D. auf Utteile anwendbar, die gegen Stderbeits= Iciftung für vorläufig bolftred́anc crllärt find? . . . . . . . .

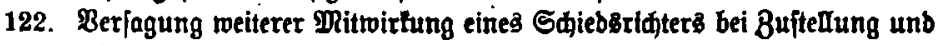

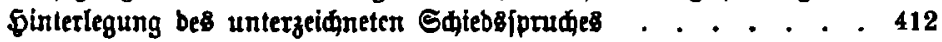

123. Feftięung bes Streitwertes, wenn mit ber Rlage bie Getausgabe ciner Qcben berfiderungapolice geforbert wirb . . . . . . . . .

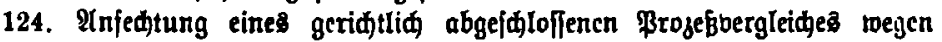

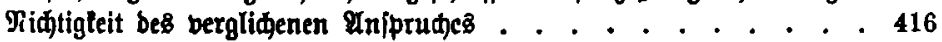

125. Pfänbung bes Redutes bes פRieters auf Benuß̧ung ber gemieteten Răumlidleitcn . . . . . . . . . . . . . . . . 
शx.

Gelte

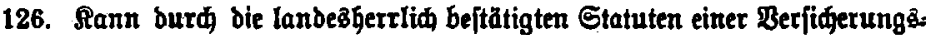

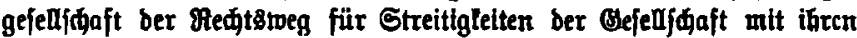

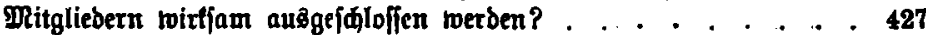

127. Эeftięung ber bei Mufbebung einet Bouftredungsmafregel bom Sdulbner zu leiftenden Simerheit. . . . . . . . .. . . . 490

Sadregifter . . . . . . . . . . . . . . . . . . . 488

Belçęstegifter . . . . . . . . . . . . . . . . . . . 451

Ghronologifife Bujammenftellung. . . . . . . . . . . . . . . 461

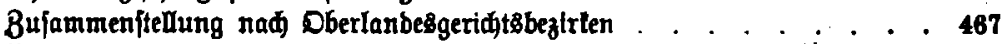

Beridtigungen . . . . . . . . . . . . . . . . . . . . 468 